Note. The above research was partially supported by the United States Air Force under Contract AF 49(638)-262.

\title{
REFERENCES
}

1. R. Courant and D. Hilbert, Methoden der mathematischen Physik, Vol. II, Springer, Berlin, 1937.

2. N. Dunford and J. Schwartz, Linear operators, Interscience, New York, 1959.

3. P. Halmos, Measure theory, Van Nostrand, New York, 1950.

4. E. Hopf, Über die Anfangswertaufgabe für die hydrodynamischen Grundgleichungen, Math. Nachr. 4 (1951), 213-231.

5. M. Riesz, Sur les ensembles compacts de fonctions sommables, Acta. Sci. Math Szeged 6 (1933), 136-142.

UNIVERSITY OF MinNESOTA

\section{ON THE BASIS PROBLEM IN NORMED SPACES ${ }^{1}$}

\section{MAHLON M. DAY}

Since Schauder's fundamental question of the paper [9]-Does every separable Banach space have a basis?-is still unanswered, we give in this note a proof that every infinite-dimensional Banach space has a closed linear subspace which has a basis with certain extra properties. Banach [1] asserts without proof the existence of a subspace with a basis. Gelbaum [4] proves the existence of a subspace which has a basis with slightly weaker extra properties than those proved in this note. The present theorem was stated, with a few words about the proof, in my book [3, p. 72], but repeated requests for copies of a paper in which proof is given suggest that that outline is too obscure.

Indeed, when this note was first sent off, the referee discovered that the proof, given there as sketched in [3], was incorrect. We give here a proof based on a generalization of the Borsuk-Ulam theorem. The other "proof" misquoted a theorem of E. Michael in an attempt to reduce the generalization to the original theorem.

THEOREM. Let $N$ be an infinite-dimensional normed space. Then there exist biorthogonal sequences $\left(b_{i}, i \in \omega\right)$ and $\left(\beta_{i}, i \in \omega\right)$ in $B$ and $B^{*}$ respectively, such that

(i) $\left(b_{i}\right)$ is a basis for the closed linear manifold $L$ in $B$ spanned by the set of all $b_{i}$.

Received by the editors April 27, 1961 and, in revised form, May 19, 1961.

1 This note was written while the author held a Senior Postdoctoral Fellowship of the National Science Foundation (U.S.A.). 
(ii) $\left\|b_{i}\right\|=\left\|\beta_{i}\right\|=1$ for all $i$ in $\omega$.

(iii) Setting $P_{m} x=\sum_{i \leq m} \beta_{i}(x) b_{i}$ for each $m$ in $\omega$, the linear operator $P_{m}$ is a projection in $L$ of norm $\leqq 1+1 / m$.

Proof. Take $b_{1}$ of norm 1 in $B$ and choose $\beta_{1}$, by the Hahn-Banach theorem, such that $\left\|\beta_{1}\right\|=\left\|b_{1}\right\|=\beta_{1}\left(b_{1}\right)=1$. If $b_{1}, \cdots, b_{m}$ in $B$ and $\beta_{1}, \cdots, \beta_{m}$, and, if necessary, certain auxiliary $\gamma_{1}, \cdots, \gamma_{k}$ in $B^{*}$ have been chosen, the choice of $b_{m+1}$ is made to depend on Borsuk's theorem in the following way:

Let $L_{m}$ be the linear hull of the $b_{i}, i \leqq m$, and let $S_{m}$ be the unit sphere $S \cap L_{m}$. If $K$ is the closed real interval $-1 \leqq t \leqq 1$, each of the sets $\beta_{i}^{-1}(K) \cap L_{m}$ and $\gamma_{j}^{-1}(K) \cap L_{m}$ contains $S_{m}$, and the intersection of these sets is a polyhedron $\Pi_{m}$ in $L_{m}$. It may happen that $\Pi_{m}$ contains only points of norm $\leqq 1+1 / m$; if this is not the case take enough elements of norm 1 in $L_{m}^{*}$, say $\alpha_{1}, \cdots, \alpha_{n}$, that $\Pi_{m}$ intersected with all the sets $\alpha_{q}^{-1}(K)$ is a polyhedron in $L_{m}$ which all lies within the sphere of radius $1+1 / m$. Let $\gamma_{k+q}$ be an element of $B^{*}$ of norm 1 which is an extension of $\alpha_{q}, q=1, \cdots, n$. Let $\Lambda_{m}$ be the (infinite-dimensional) intersection of the hyperplanes $\beta_{i}^{-1}(0), i \leqq m$, and $\gamma_{j}^{-1}(0), j \leqq k+n$.

In $\Lambda_{m}$ choose any $(m+1)$-dimensional subspace $\Lambda_{m}^{\prime}$ and in $\Lambda_{m}^{\prime}$ consider the unit sphere $S_{m}^{\prime}=S \cap \Lambda_{m}^{\prime}$. Define a mapping $\phi$ from $S_{m}^{\prime}$ into convex compact subsets of $L_{m}$ by

$$
\phi\left(x^{\prime}\right)=\left\{y: y \in L_{m} \text { and }\left\|x^{\prime}+y\right\|=\left\|x^{\prime}+L_{m}\right\|\right\}
$$

where, as usual,

$$
\left\|x^{\prime}+L_{m}\right\|=\inf \left\{\left\|x^{\prime}+z\right\|: z \in L_{m}\right\} .
$$

Then $\phi(-x)=-\phi(x)$ and $\phi$ is upper semicontinuous; that is, if $x_{n}^{\prime}$ tends to $x^{\prime}$, if $y_{n} \in \phi\left(x_{n}^{\prime}\right)$, and $y=\lim _{n \in \omega} y_{n}$ exists, then $y \in \phi\left(x^{\prime}\right)$. Also $\|y\| \leqq 1+1 / m$ if $y \in \phi(x)$.

To prove that an $x$ exists in $S_{m}^{\prime}$ such that $0 \in \phi(x)$, we generalize the Borsuk-Ulam theorem in precisely the same way that Kakutani [7] generalized the Brouwer fixed-point theorem. Jaworowski [6] has a generalization of Borsuk's Theorems I and II which implies this (his $\phi(x)$ is acyclic compact) but rather than appeal to that homological proof we give here a direct proof by approximation using the Borsuk-Ulam theorem for most of the work.

THEOREM. Let $E$ be a compact convex subset of $L_{m}$ and let $\phi$ be a function defined on $S_{m}^{\prime}$ such that, for each $x, \phi(x)$ is a closed convex subset of $E$. Assume that $\phi$ is upper semicontinuous (or equivalently, that the graph of $\phi$ is closed) and that $\phi$ is antipodal; that is, that $\phi(-x)=-\phi(x)$. Then there is an $x$ in $S_{m}^{\prime}$ such that $0 \in \phi(x)$. 
Let $\Sigma_{n}$ be a sequence of "simplicial" decompositions of $S_{m}^{\prime}$ into "spherical triangles" with the diameters of the simplexes of $\Sigma_{n}$ tending to zero as $n \rightarrow \infty$, and let $x_{n i} \cdots x_{n k}$ be the vertices of $\Sigma_{n}$ in some order. Define a continuous function $\phi_{n}$ from $S_{m}^{\prime}$ into $E$ by first choosing $\phi_{n}\left(x_{n i}\right)$ as an element of $\phi\left(x_{n i}\right)$, choosing $\phi_{n}\left(-x_{n i}\right)=-\phi_{n}\left(x_{n i}\right)$, and then extending the map to all $S_{m}^{\prime}$ simplicially. Each $\phi_{n}$ is continuous and antipodal, so the Borsuk-Ulam theorem asserts that there is a point $p_{n}$ of $S_{m}^{\prime}$ such that $\phi_{n}\left(p_{n}\right)=0$.

(In [2, Theorem II] it is asserted only that some pair of antipodal points is carried to a single image point; but $\phi_{n}$ maps antipodal pairs to pairs symmetric about 0 ; these can coincide if and only if they both are zero.)

$p_{n}$ is in some simplex of $\Sigma_{n}$ with vertices $y_{n 0}, \cdots, y_{n m}$, so $p_{n}=\sum_{i=0}^{m} \lambda_{n i} y_{n i}$, where $\lambda_{n i} \geqq 0, \quad \sum_{i} \lambda_{n i} \geqq 1$, and $\lim _{n} \sum_{i} \lambda_{n i}=1$. Choose a subsequence $\left\{n_{j}\right\}$ of the integers so that $y_{i}=\lim _{j} y_{n_{j} i}$ exists, $z_{i}=\lim _{j} \phi_{n_{j}}\left(y_{n_{j} i}\right)$ exists, and $\lambda_{i}=\lim _{j} \lambda_{n_{j i}}$ exists for $i=0,1, \cdots, m$. Then $p=\lim _{j} p_{n_{j}}$ exists also and $p=y_{i}$ for all $i$. Also $\sum_{i} \lambda_{i}=1$. Now

$$
0=\phi_{n_{j}}\left(p_{n_{j}}\right)=\phi_{n_{j}}\left(\sum_{i} \lambda_{n_{j} i} y_{n_{j} i}\right)=\sum_{i} \lambda_{n_{j} i} \phi_{n_{j}}\left(y_{n_{j} i}\right) \rightarrow \sum_{i} \lambda_{i} z_{i} .
$$

But $z_{i} \in \phi(p)$ because $\phi$ is upper semicontinuous. Hence $0=\sum \lambda_{i} z_{i}$ $\in \phi(p)$ because $\phi(p)$ is convex.

To return now to the main theorem, let $b_{m+1}$ be any point of $S_{m}^{\prime}$ such that $0 \in \phi\left(b_{m+1}\right)$. Let $\beta_{m+1}$ in $B^{*}$ be chosen so that it is of norm 1 , vanishes on the $b_{i}, i \leqq m$, and is 1 at $b_{m+1}$. This is possible because the calculation with Borsuk's theorem arranged matters so that in $L_{m+1}$ the projection along $L_{m}$ onto the line through $b_{m+1}$ is of norm 1. Also $\Lambda_{m}$ was chosen so that the projection of $\Lambda_{m}+L_{m}$ along $\Lambda_{m}$ onto $L_{m}$ is of norm $\leqq 1+1 / m$. This induction process defines sequences $\left(b_{i}\right)$ and $\left(\beta_{i}\right)$.

If $L^{\prime}$ is the union of all the $L_{m}$, then for each $m$ the function $P_{m}$ is defined in $B$ and has in $L^{\prime}$ norm $\leqq 1+1 / m$, because all the $b_{i}, i>m$, are in $\Lambda_{m}^{\prime}$. Since $L^{\prime}$ is dense in $L,\left\|P_{m}\right\|_{L} \leqq 1+1 / m$. The set of those $x$ in $L$ where $\lim _{m \in \omega} P_{m} x=x$ includes all of the $b_{i}$ and is closed in $L$ $[1$, p. 79 , Theorem 3$]$, so it is all of $L$; that is, $\left(b_{i}\right)$ is a basis for $L$, and the proof is complete.

Note that if in (iii) the condition $\left\|P_{m}\right\| \leqq 1+1 / m$ were replaced by $\left\|P_{m}\right\| \leqq 1+c_{m}$, where $\left(c_{m}\right)$ is any preassigned bounded sequence of positive numbers, the proof would be almost unaltered.

It may be noted that if this process is applied to $m(\omega)$, the space of all bounded real sequences, nothing in the process prevents the choice of $b_{n}$ equal to the usual $n$th basis vector in $c_{0}(\omega)$, the subspace of sequences converging to zero; that is, $b_{n}(n)=1, b_{n}(m)=0$ if $m \neq n$. Then the corresponding sequence of $\beta$ 's may be total over $m(\omega)$, and 
the process stops even though $m(\omega)$ is not separable. This example shows that the basis found need not be boundedly complete in the sense of James [5] and Karlin [8]. It also shows, by Sobczyk's result of [10], that there need not be any continuous projection of $N$ onto $L$. The construction of the theorem if applied in $l^{1}(\omega)$ might also yield the usual basis, which shows that the biorthogonal system $\left\{\beta_{i}\right\}$ need not be a basis for $L^{*}$.

In view of Karlin's theorem that there is no unconditional basis in $C[0,1]$, the space of real-valued functions continuous on the closed unit interval, it would be of interest to improve this construction to show that every $B$ contains an $L$ with an unconditional basis; but whether this is possible is not known.

\section{BiBLIOGRAPHY}

1. S. Banach, Theorie des operations lineaires, Monogr. Mat., Vol. 1, Warsaw, 1932.

2. K. Borsuk, Drei Sätze über die n-dimensional Euklidische Sphäre, Fund. Math. 20 (1933), 177-190.

3. M. M. Day, Normed linear spaces, Springer, Berlin-Göttingen-Heidelberg, 1958.

4. B. R. Gelbaum, Notes on Banach spaces and bases, An. Acad. Brasil. Ci. 30 (1958), 29-36.

5. R. C. James, Bases and reflexivity of Banach spaces, Ann. of Math. (2) 52 (1950), 518-527.

6. J. W. Jaworowski, Theorem on antipodes for multi-valued mappings and a fixed point theorem, Bull. Acad. Polon. Sci. Cl. III, 4 (1956), 187-192.

7. S. Kakutani, A generalization of Brouwer's fixed point theorem, Duke Math. J. 8 (1941), 457-459.

8. S. Karlin, Bases in Banach spaces, Duke Math. J. 15 (1948), 971-985.

9. J. Schauder, Zur Theorie stetiger Abbildungen in Funktionalräumen, Math. Z. 26 (1927), 47-65 and 417-431.

10. A. Sobczyk, Projection of the space $m$ on its subspace $c_{0}$. Bull. Amer. Math. Soc. 47 (1941), 938-947.

UNIVERSITY OF ILLINOIS 